This document is the unedited Author's version of a Submitted Work that was subsequently accepted for publication in the Journal of the American Chemical Society, copyright $\odot$ American Chemical Society, after peer review. To access the final edited and published work see http://dx.doi.org/10.1021/acsnano.5b06931 


\section{Nanoscale study of polymer dynamics}

Masoumeh Keshavarz ${ }^{1,2^{*}}$, Hans Engelkamp ${ }^{1 *}$, Jialiang $\mathrm{Xu}^{2^{*}}$, Els Braeken ${ }^{3}$, Matthijs B. J. Otten $^{2}$, Hiroshi Uji-i ${ }^{3}$, Erik Schwartz ${ }^{2}$, Matthieu Koepf ${ }^{2}$, Anja Vananroye ${ }^{3}$, Jan Vermant ${ }^{4,5}$, Roeland J. M. Nolte ${ }^{2}$, Frans C. De Schryver ${ }^{4}$, Jan Kees Maan ${ }^{1}$, Johan Hofkens ${ }^{4,5}{ }^{*}$, Peter C. M. Christianen ${ }^{1}$, Alan E. Rowan ${ }^{2 *}$

${ }^{1}$ High Field Magnet Laboratory, Institute for Molecules and Materials, Radboud University Nijmegen, Toernooiveld 7, NL-6525 ED Nijmegen, the Netherlands.

${ }^{2}$ Radboud University Nijmegen, Institute for Molecules and Materials, Department of Molecular materials, Heyendaalseweg 135, 6525 AJ Nijmegen, The Netherlands.

${ }^{3}$ Division of Molecular Imaging and Photonics, Department of Chemistry, Katholieke Universiteit Leuven, Clestijnenlaan 200 F, B-3001 Heverlee, Belgium

${ }^{4}$ Department of Chemical Engineering, Katholieke Universiteit Leuven, de Croylaan 46, B-3001 Heverlee, Belgium

${ }^{5}$ ETH Zürich, Department of Materials - Hönggerberg, Wolfgang-Pauli-Strasse 10, CH-8093 Zürich, Switzerland

*EMAIL ADDRESSES: m.keshavarz@science.ru.nl; h.engelkamp@science.ru.nl; j.xu@science.ru.nl; johan.hofkens@chem.kuleuven.be; a.rowan@science.ru.nl 
The thermal motion of long and stiff polymers in a crowded environment is anisotropic and highly confined. Understanding the fundamental dynamics of such systems remains an obstacle despite theoretical and experimental studies for more than thirty years. Here we describe the dynamics of extremely stiff synthetic polymers using time-resolved single molecule fluorescence microscopy and demonstrate that individual chains display tube-like motion, as predicted by the theory of De Gennes, Doi, and Edwards. We show that in one experiment all characteristic time constants and length scales including their distributions in heterogeneous systems can be obtained, providing a detailed understanding of polymer dynamics at the single chain level.

The rheology of polymers in melts and solution that arises from chain entanglement effects has been unveiled by the reptation model, which was proposed by de Gennes $\stackrel{1}{-}$ and extended by Edwards and Doi 2. In this model a polymer chain is confined by the surrounding matrix, and moves inside an imaginary tube defined by the transient network of entangled neighbouring chains. The polymer molecule cannot make transverse movements, and may relax only along the tube in a snake-like fashion (reptation). Reptation occurs if several criteria are fulfilled: for instance, the polymer chain should exceed a critical length, which depends on the system under investigation. Furthermore, the concentration of the polymer solution should be larger than the critical concentration at which entanglement starts to play a role.

The fundamental work on reptation theory has stimulated both theoretical and experimental studies to investigate the tube-like motion (reptation) in entangled media. Several experimental techniques such as

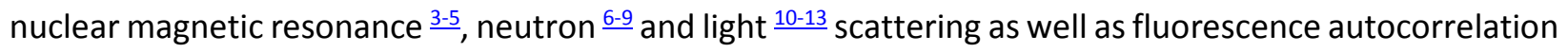
spectroscopy $\stackrel{14-17}{ }$ have been used to probe the dynamics of entangled polymers in bulk. Texeira et al $\underline{18}$ studied the dynamics of individual entangled DNA chains under the effect of a bulk flow deformation. For different chains in the same flow experiment very different behaviour, which is evidence for molecular individualism, was found. 
Perkins and co-workers $\stackrel{19}{\underline{1}}$ were the first to directly observe reptation using optical microscopy. They followed the relaxation of a labelled individual DNA chain after stretching it with optical tweezers. It was shown that the only way the polymer chain could relax was by following its own path without making transversal movements. Käs and co-workers $\underline{20}$ followed the motion of labelled actin filaments and imaged the confining tube in which the polymer chain could move by superimposing a large number of images and plotting the contours of the observed polymer chains. The restricted motion could be seen as undulations of the actin filament in a tube formed by its neighbours. The tube diameter was determined and was shown to decrease with increasing concentration. Further studies on actin filaments and DNA chains have been published in more recent years $\frac{21-26}{}$. Also a study on the motion of synthetic single-walled carbon nano tubes in a gel, aimed at understanding the effect of flexibility on the diffusion of stiff filaments under confinement, has appeared $\underline{27}$.

Sofar, the direct observation of the reptation behaviour of completely synthetic polymers by single molecule techniques has not yet been reported in the literature. In this work we present a comprehensive study on the dynamics of extremely stiff synthetic polymers in concentrated polymer solutions at the single-chain level by means of fluorescence microscopy in both space and time. We show that in a single experiment all relevant length scales and time constants can be accessed. Furthermore, we probed the dynamics of the individual polymer chains in their longitudinal relaxing modes and demonstrate the validity of the scaling laws in reptation theory at the single chain level.

\section{Results and Discussion}

The synthetic polymer that we used in our study is a polyisocyanopeptide that can be synthesized by a nickel(II) catalyzed polymerization reaction from an isocyanopeptide $\stackrel{28}{ }$. The polymer molecules have a rigid helical backbone with approximately 4 monomer units per helical turn. In our approach, we directly visualized the reptation motion of a polyisocyanopeptide, by tracking the motion of a perylene labelled 
derivative (FP), poly(isocyano-L-alanine propanylperylene) (L-PIAP), in a matrix of unlabelled poly (isocyano-L-alanyl-D-alanine methyl ester) (L,D-PIAA)molecules $\stackrel{29}{\underline{29}}$ using wide-field microscopy. The high molecular weight and the rigidity of a polyisocyanopeptide, which can be unusually long (up to $20 \mu \mathrm{m}$ )

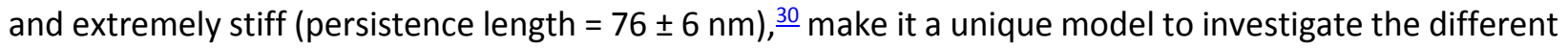
aspects of polymer chain dynamics in an in-depth single molecule study. This type of polymer has been

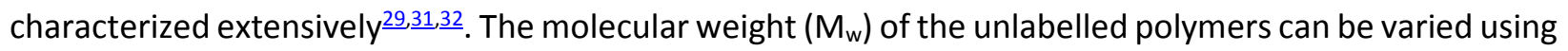
different ratios of nickel (II) initiator to monomer. In our study we used ratios of [initiator]/[monomer] = $1 / 10,000$ and $1 / 2,000$, yielding polymers with an average length of $400 \mathrm{~nm}$.

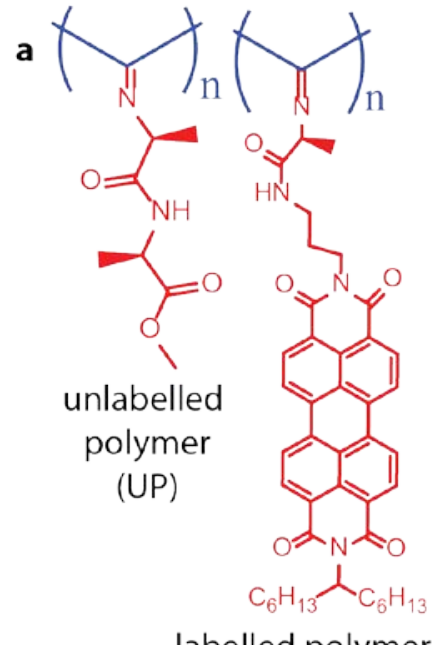

labelled polymer

(FP)
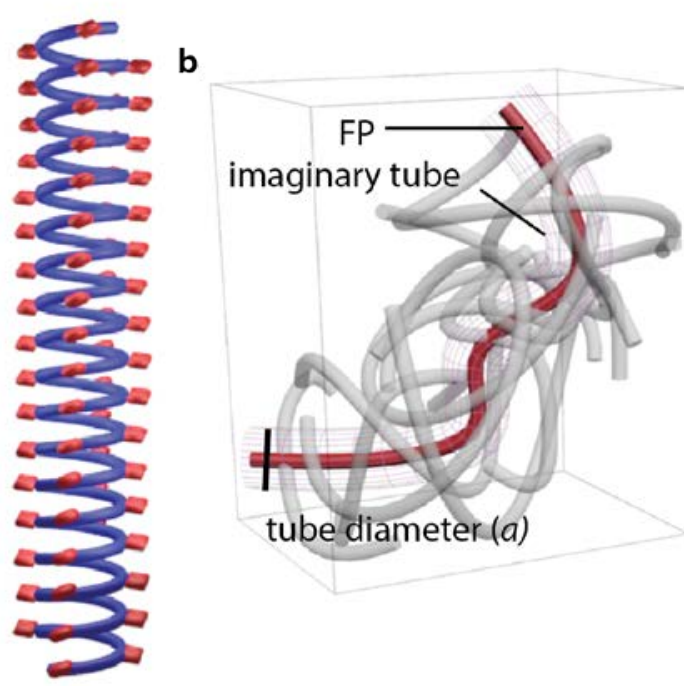

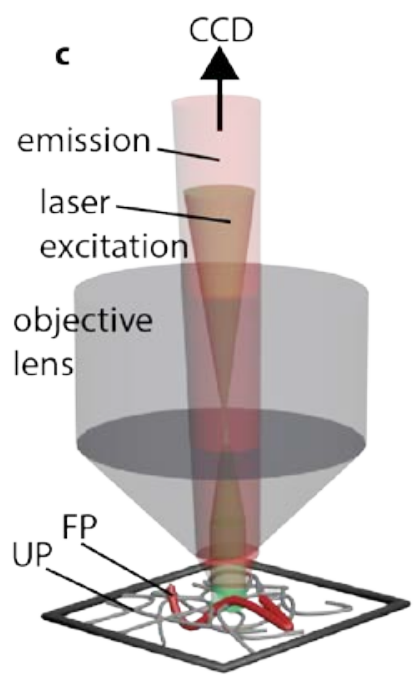

Figure 1| Schematic representation of the single polymer reptation experiment. a, Chemical structure of unlabelled L,D-PIAA (UP) and labelled L-PIAP with perylenediimide as chromophore (FP) and cartoon of the helical structure of a polyisocyanide (backbone in blue, side groups in red). b, 3D presentation of the reptation. A fluorescently labelled polymer, FP (red line), moves within an imaginary tube (wireframe purple tube with diameter $a$ ) formed by the entangled neighbouring unlabelled polymer chains, UP, (the grey lines). c, Schematic representation of the experiment. The labelled polymer (FP, red lines) in a transparent matrix of unlabelled polymers (UP, grey lines) was excited with a laser (green light). The fluorescence emission (red light) was collected by an objective lens and imaged on a CCD camera. 
The basic molecular structures of the unlabelled and labelled polyisocyanide polymers (backbone in blue, side groups in red) are shown in figure 1a. The polymer helical conformation is stabilized by hydrogen bonds between the amide groups of the side chains $\underline{29}$. Details of the characterization of the polymers can be found in the Materials Section.

The studied tube motion in three dimensions is illustrated in figure $1 \mathrm{~b}$, where a labelled polymer (FP, red line) moves within an imaginary tube (the purple wireframe tube with diameter $a$ ) characterized by its tube diameter defined by the surrounding polymer chains (UP, grey lines). The diameter of the tube, $a$, corresponds to the average distance between the nearest entanglement points $\stackrel{1}{=}$.

The tube motion of the labelled polymer, FP, within the unlabelled ones, UPs, was directly visualized using a wide-field fluorescence microscopy set-up (Fig. 1c). The sample containing mainly unlabelled polymers and only a few labelled ones was photo-excited with a laser $(\lambda=543 \mathrm{~nm})$. The fluorescence (emission) was collected through an objective lens and imaged with a CCD camera. Movies with predominantly 2D motion were selected (> 20 movies out of 100) for further analysis. These movies were analyzed using home-made software, as exemplified in figure 2. Each raw frame (Fig. 2a) was analyzed individually. First, a background, which was calculated using a 51 x 51-kernel median filter, was subtracted and the image was smoothed using a $5 \times 5$-kernel Gaussian filter. Using $5 \times 5$-Kernel turned out to yield the best consistent fit. The resulting picture (Fig. 2b) was then fitted with 2D Gaussians (Fig. 2c). The coordinates of Gaussians belonging to a single polymer chain were grouped together, spline-interpolated, and used for further analysis. Using this procedure, we obtained, as a function of time, the end-to-end distance, contour length $(L)$, centre of mass position and motion, and the middle point of the polymer chain (Fig. 2d). This method permits us to study the polymer motion as a function of the properties of the reptating polymer, most importantly the contour length, and the characteristics of the surrounding matrix, which is only possible in a single molecule experiment. To this end, we calculated the perpendicular $\left(d_{\perp}\right)$ and 
longitudinal displacements $\left(d_{\|}\right)$from the movies, as shown in figure 2e. The perpendicular displacement in time (related to the tube diameter, see below) was calculated as the distance between the middlepoint of the chain in frame $t_{2}$ to the nearest point of the chain in frame $t_{1}$, for all pairs of frames in a movie. The longitudinal displacement (related to forward motion) was then calculated from the centerto-center displacement and Pythagoras' theorem.
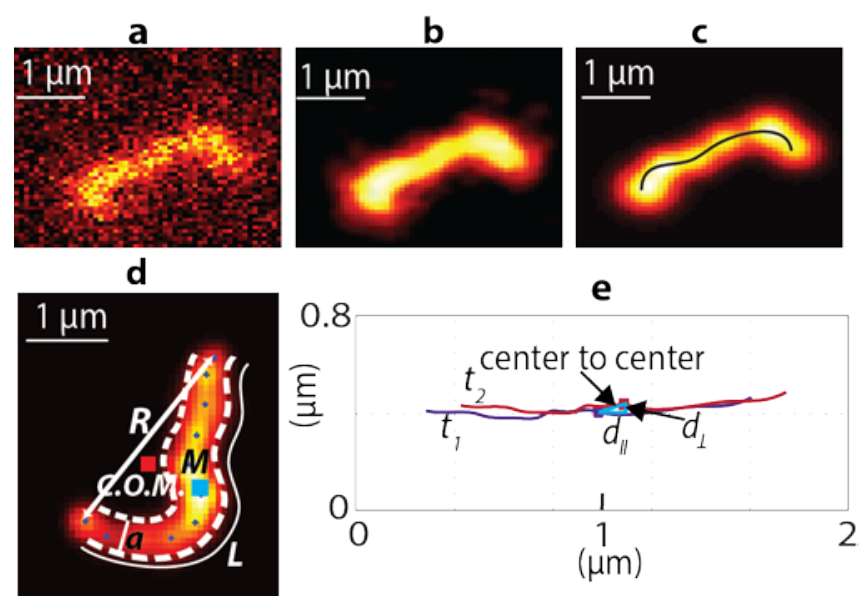

Figure 2| Data analysis. a,b, An example of a raw, and a background subtracted, smoothed fluorescence image. c, Reconstructed image from the fitting procedure and the chain conformation (the black line in the image). $\mathbf{d}$, The parameters extracted from the fitting procedure, such as end-to-end distance $(R)$, centre of mass (C.O.M.), middle point of the contour $(M)$, the contour length $(L)$ and the tube diameter $(a)$. The points along the polymer chain indicate the fitted Gaussians. e, $d_{\perp}$ between the chain at $t_{1}$ and $t_{2}$ is calculated as the smallest distance between the curve at $t_{1}$ and $M$ at $t_{2} ; d_{\|}$is calculated using the centreto-centre displacement and $d_{\perp}$. 


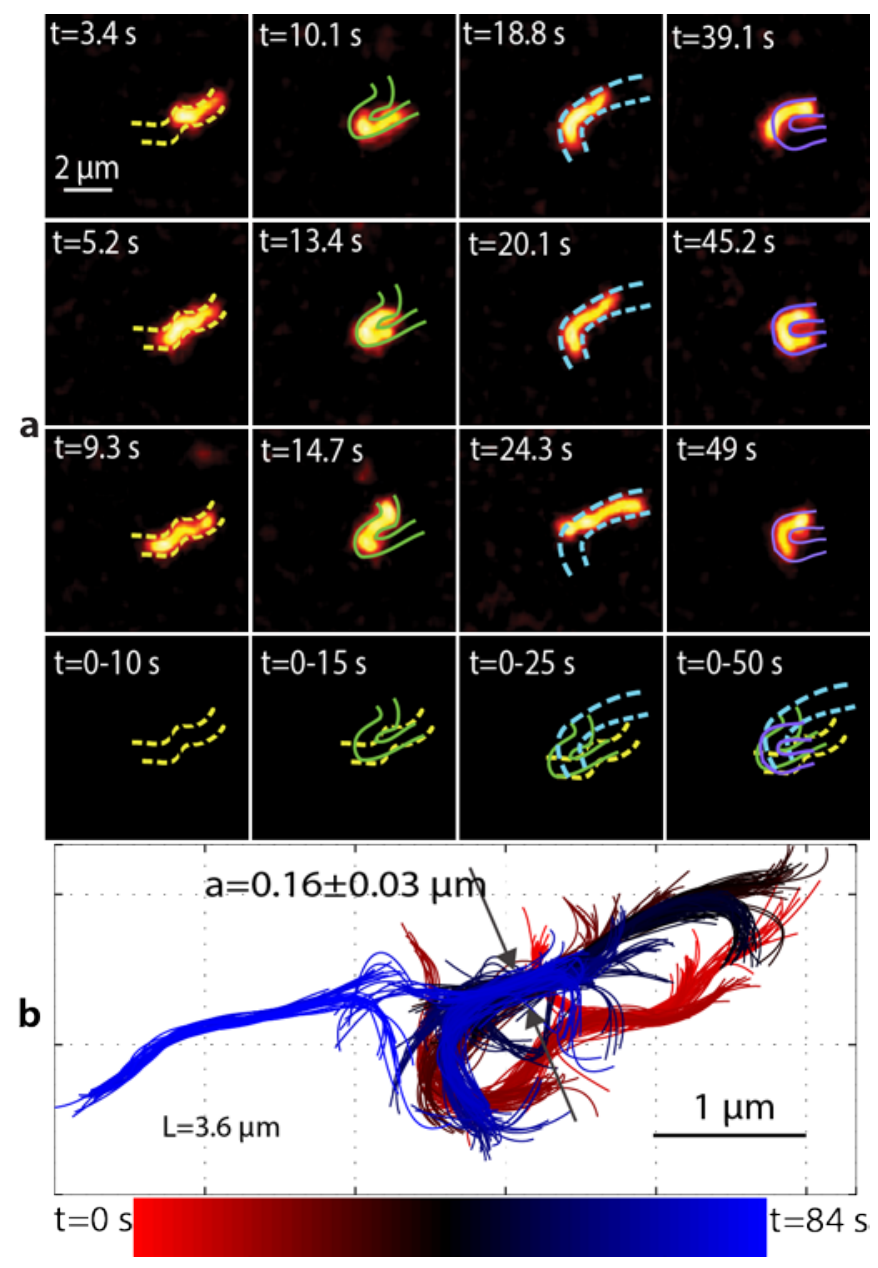

Figure 3| Movie snapshots showing the reptation motion (movie \#1, S.I.). a, Snapshots of a labelled LPIAP (FP) chain in a concentrated solution of unlabelled L,D-PIAA (UP). The overall tube was obtained by superposition of the snapshots corresponding to the timescales indicated. In the second column (10-15 s), the polymer molecule started to escape the yellow tube (0-9 s) represented by the dashed lines in the first column since the new tube (green solid line in the second column) did not follow the same path of the original tube. The tube escape can be seen in all the following images. $\mathbf{b}$, The superimposed conformations of the labelled polymer for the entire movie from $t=0$ to $t=84 \mathrm{~s}$. The colour code indicates the time. The tube diameter was extracted to be $a=160 \pm 10 \mathrm{~nm}$ and the disentanglement time (the time where all overlap with the previous conformation has vanished) of the first tube motion (the $U$ shape in the right part of the figure) was estimated to be $\tau_{d}=50 \mathrm{~s}$.

Figure 3a shows representative snapshots of a movie recorded from a labelled L-PIAP polymer chain (FP) in a concentrated solution of unlabelled L,D-PIAA (UP) molecules. It is clearly visible that the labelled polymer chain is moving in an imaginary tube (indicated with lines next to the chain) formed by its 
unlabelled neighbours (see also movie\#1 in the S.I). The overall tube in which the single polymer molecule is moving for short time periods is shown in the lower part of the figure. It is obtained by superimposing the snapshots corresponding to the timescales indicated. For example, in the second column (10-15 s), it can be seen that the polymer has started to escape the yellow tube (0-9 s) represented by the dashed lines in the first column since the new tube (green lines in the second column) does not follow completely the same path of the original tube. This tube escape can be seen in all the following images as the surrounding polymers that make the tube are dynamic. Looking at the snapshots it is evident that after about $50 \mathrm{~s}$ the polymer chain has left its original tube completely. In figure $3 \mathrm{~b}$ we have plotted the superimposed conformations of the labelled polymer for the entire movie from $t=0$ to $t=84 \mathrm{~s}$ and the tube diameter is estimated to be $a=160 \pm 10 \mathrm{~nm}$. The imaginary tubes in figure 3a are clearly visible in figure $3 \mathrm{~b}$. It can be seen that the blue lines (the most left part of Fig. 3b) define a completely different tube and, thus, the polymer chain has left the original tube (the $U$ shaped tube in the right part of Fig. $3 b$ ) There is no overlap between the most left lines in the figure and the first tube (the $U$ shaped lines in the right part of the Fig. 3b). This gives an approximation of the disentanglement time for the first tube, which is estimated to be $\tau_{d}=50 \mathrm{~s}$. Additional data is provided in the Supplementary Information.

The polymer dynamics is found to be strongly dependent on the concentration of the surrounding matrix, UP (Fig. 4). The inset of figure 4a displays the FP polymer motion within a dilute UP polymer matrix, (concentration $=3 \mathrm{mg} / \mathrm{ml})$. In a period of $80 \mathrm{~s}$ the polymer chain explores a rather wide area $\left(4 \times 4 \mu \mathrm{m}^{2}\right.$ ) in a diffusive manner typical for Brownian motion; in contrast, a FP polymer in a more dense UP matrix (concentration $=5 \mathrm{mg} / \mathrm{ml}$, Fig. $4 \mathrm{~b}$ inset) is restricted by the neighbouring chains limiting the lateral motion with respect to the tube. The tube diameter $(a=0.16 \pm 0.03 \mu \mathrm{m})$ can be estimated from this image (arrows in the inset of Fig. 4b), but also by plotting a histogram of the perpendicular displacements $\left(d_{\perp}\right.$, solid symbols, Fig. 4b). The latter data revealed a very narrow Gaussian distribution (solid lines in Fig. 4b) the width of which corresponds to a tube diameter of $a=0.15 \pm 0.03 \mu \mathrm{m}$. The $d_{\perp}$ histogram of the dilute 
matrix showed a very broad peak (symbols in Fig. 4a) with a width of $a=1.00 \pm 0.03 \mu \mathrm{m}$, one order of magnitude larger than the tube diameter in the concentrated matrix (see movies \#2 and \#3 in S.I.). The persistence length, $l_{p}$, and contour length, $L$, of the labelled polymer also can be calculated from our experimental data (see S. I.).
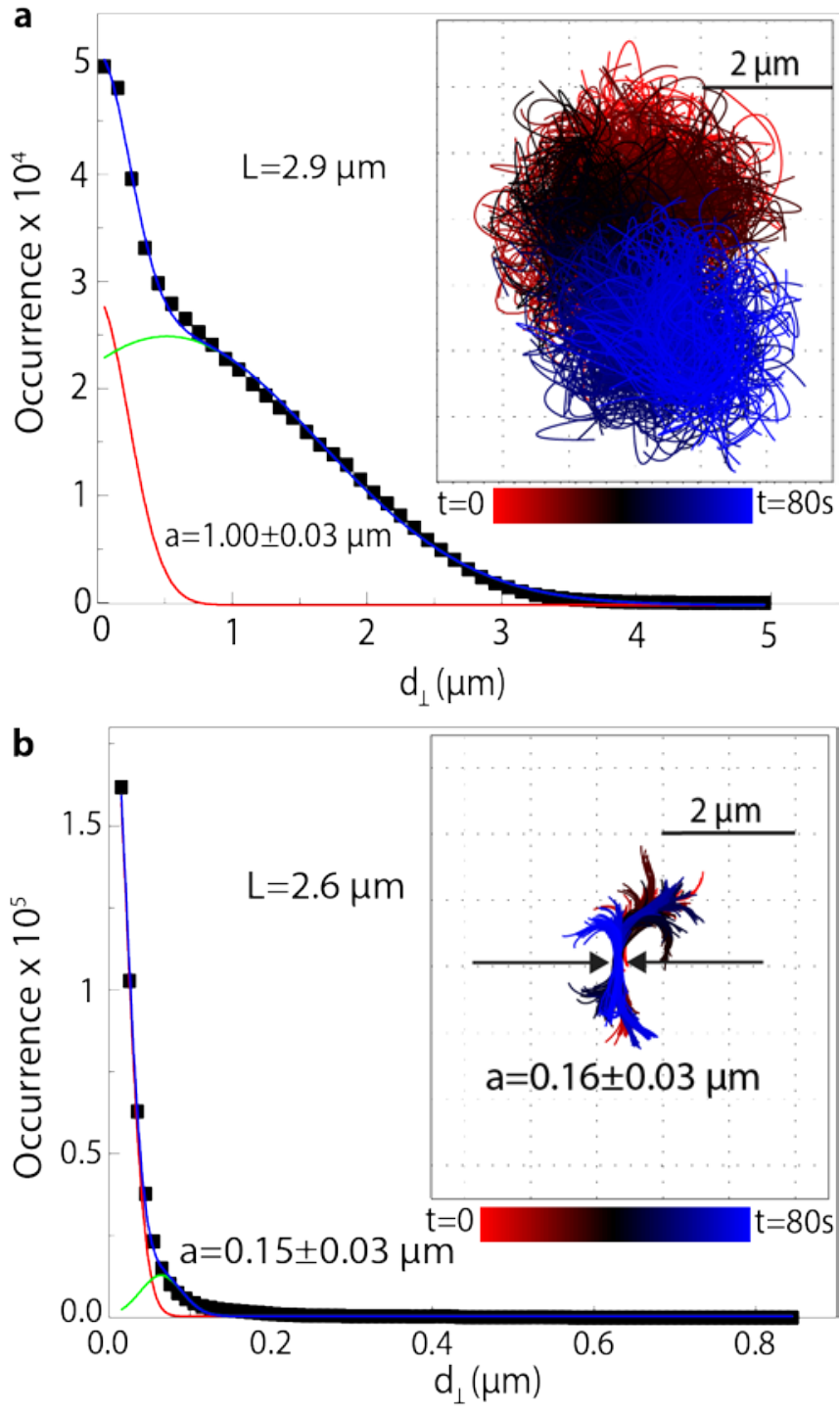

Figure 4| Polymer motion in dilute (upper panel) and concentrated (lower panel) solutions. Insets (both have the same scale) show: a, the polymer motion in a dilute matrix (concentration $=3 \mathrm{mg} / \mathrm{ml}$ ) during a time of $t=80 \mathrm{~s}$. The polymer chain explores a large area representing Brownian motion, $\mathbf{b}$, the polymer motion in a concentrated matrix (concentration $=5 \mathrm{mg} / \mathrm{ml}$ ), which by contrast represents a confined motion referred to as reptation with a tube diameter of $a=0.16 \pm 0.03 \mu \mathrm{m}$ (indicated by arrows in the figure). The occurrence of perpendicular displacements, $d_{\perp}$, (solid symbols in Figs. a and b) is indicated. A 
narrow Gaussian distribution (solid lines in Fig. 4b) is observed for the concentrated UP matrix, of which the width corresponds to the tube diameter $(a=0.15 \pm 0.03 \mu \mathrm{m})$, while the dilute matrix shows a broad peak (symbols in Fig. 4a) with a width of $a=1.00 \pm 0.03 \mu \mathrm{m}$.

Our movies unambiguously demonstrate that the polymer molecules display constrained motion. Moreover, from the data we can obtain detailed and quantitative information about the space- and timedependence of the polymer dynamics. This information becomes apparent when the mean value of perpendicular displacement, $\left\langle d_{\perp}\right\rangle$, and the mean square value of longitudinal displacement, $\left\langle d_{\|}^{2}\right\rangle$, as a function of the lag time for a singly labelled chain in a concentrated matrix, is calculated. As an example, we considered a situation, in which the polymer chain changes its original tube completely several times within 80 seconds of its motion (Fig. 5a). In order to calculate the tube diameter of this chain, we divided the movie (movie\#4 see S.I.) into four parts, such that each part contains at least one tube. The perpendicular displacement, $d_{\perp}$, was averaged for each lag time and is plotted in figure 5b (1-4), along with $\left\langle d_{\perp}\right\rangle$ for the entire movie (Fig. $\left.5 b(5)\right)$. We observed two regimes where $\left\langle d_{\perp}\right\rangle$ follows a power law $\underline{33}$. The slope of the fits changes from $1 / 4$ to $1 / 2$ in each plot. This change is the point where the polymer segments face tube constraints and transition from local reptation to reptation occurs. The average perpendicular displacement in the crossing point corresponds to the tube diameter, which is extracted for 1 to 4 and the entire movie, 5 (Fig. $5 c$ ). The average value is the same, $a=0.08 \pm 0.02 \mu \mathrm{m}$, for all parts of the movie highlighting the remarkable homogeneity of the matrix over the studied area. We emphasize that with this method we can probe directly the local homogeneity or the heterogeneity of the matrix by tracking the tube diameter of the labelled single polymer over time. To obtain information about the dynamics of the reptation motion of individual chains we plotted the mean square value of longitudinal displacement, $\left\langle d_{\|}^{2}\right\rangle$, $\left(\left\langle d_{\|}^{2}\right\rangle\right.$ was calculated using same procedure as $\left.\left\langle d_{\perp}\right\rangle\right)$ versus lag time, figure $5 \mathrm{~d}$. The reptation model predicts four dynamical regimes for segment diffusion. They are summarized below $\stackrel{34}{ }$ : 
(i) $\quad t<\tau_{e}$ : In this regime, the chain explores the maximum of its lateral displacement and does not feel topological constrictions. The timescale on which the defect propagates along the chain is referred to as entanglement time $\tau_{e}$. In this regime, the mean square longitudinal displacement scales with time as $\left\langle d_{\|}^{2}\right\rangle \propto t^{1 / 2}$, where $\left\langle d_{\|}^{2}\right\rangle$ is the mean square longitudinal displacement of the chain segments. Though this time scale is very small we have access to it indirectly within our experimental approach.

(ii) $\quad \tau_{e}<t<\tau_{R}$ : this regime is referred to as local reptation and $\left\langle d_{\|}^{2}\right\rangle \propto t^{1 / 4}$. The polymer diffuses over a distance of the order of its size during a characteristic time called the Rouse time $\tau_{R}$.

(iii) $\quad \tau_{R}<t<\tau_{d}$ : this is the regime of reptation where the longitudinal creep through the tube is possible and $\left\langle d_{\|}^{2}\right\rangle \propto t^{1 / 2}$. The parameter $\tau_{d}$, the disentanglement time, is the time required for one chain to move out of its original tube.

(iv) For times longer than $\tau_{d}$, normal diffusive behaviour takes place and $\left\langle d_{\|}^{2}\right\rangle \propto t$.

In figure $5 d$ we observe two regimes (the solid lines separated with a dashed line in Fig. $5 d$ ) where $\left\langle d_{\|}^{2}\right\rangle$ relates in a power law to the lag time with an exponent of $0.28 \pm 0.05$, the first referring to the local reptation and the second with exponent of $0.5 \pm 0.05$ to the reptation regime. The crossing point of these two regimes is the Rouse time, $\tau_{R}=0.4 \pm 0.1 \mathrm{~s}$, for a reptating chain within the single molecule approach in agreement with numerical simulations $\frac{33}{3}$. To the best of our knowledge, our result is the first experimental observation of the specific time dynamics of polymers within the Rouse (ii) and reptation (iii) regimes. Due to photo-bleaching of the labelled polymer, it was difficult to obtain enough data points to determine the disentanglement time, $\tau_{d}$, from this plot. The time resolution is limited in our experimental setup to $0.1 \mathrm{~s}$ and does not allow us to directly probe the processes faster than the entanglement time. Occasionally, in a small percentage of our real-time data the reptation time was longer than the measurement time and we were not able to observe the reptation regime (see S.I.). 

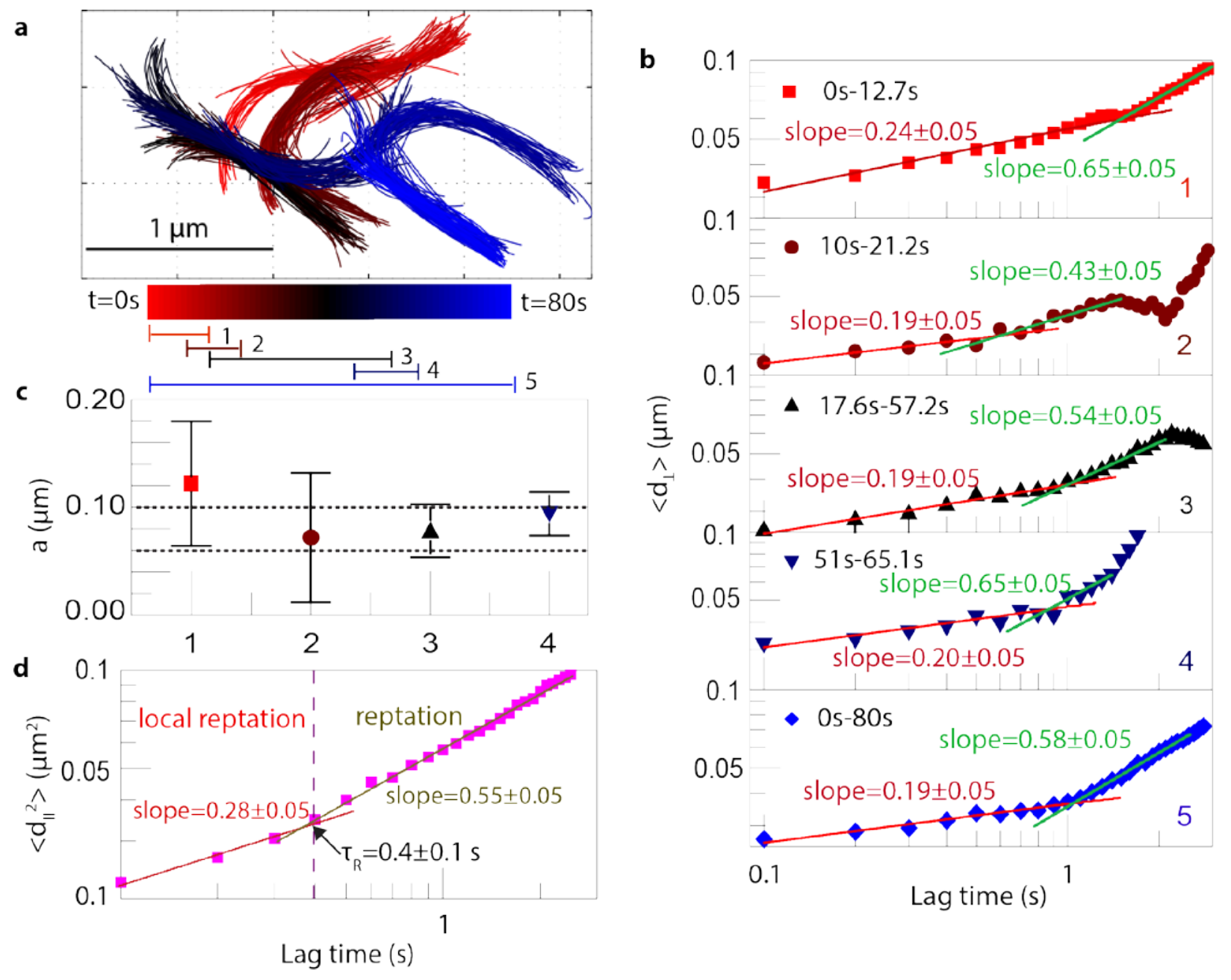

Figure 5| Space- and time-dependence of the polymer motion. a, The motion of a labelled FP polymer chain in the concentrated UP matrix where the polymer chain changes its path several times within 80s. The colour bar shows the time. This movie is divided into four parts (solid lines below the figure). $\mathbf{b},\left\langle d_{\perp}\right\rangle$ versus lag time for all parts of the movie, 1 to 4 , and for the entire movie, 5 . The average value of perpendicular displacement at the crossing point (which we define as the point where the slope of the fitted lines changes from $1 / 4$ to $1 / 2$ ) gives an estimate of tube radius, $a / 2$. c, The tube diameter for movie parts 1 to 4 and the entire movie 5 versus plot numbers (the dashed lines is the tube diameter of the entire movie) indicating the homogeneity of the matrix over this period. $\mathbf{d},\left\langle d_{\|}^{2}\right\rangle$ as a function of lag time, indicating the regimes of local reptation (solid line with a slope of $0.28 \pm 0.03$ ) and reptation (solid line with a slope of $0.55 \pm 0.04$ ). The time point between the two regimes is the Rouse time $\tau_{R}=0.4 \pm 0.1 \mathrm{~s}$.

In order to link our single molecule data to bulk polymer properties, we have used the parameters $\left(\tau_{d}, \tau_{R}\right.$ and a) to explore the analytic expressions for time- and space-dependent behaviour of entangled polymers at the single chain level. The Rouse time, $\tau_{R}$, disentanglement time, $\tau_{d}$, and entanglement time, 
$\tau_{e}$, are related by $\stackrel{35}{\text { : }} \tau_{R}=Z^{2} \tau_{e}$ and $\tau_{d}=3 Z^{3} \tau_{e}$ thus, $\tau_{d}=3 Z \tau_{R}$ where $Z$ is defined as the number of entanglements per chain. The relationship between disentanglement time and Rouse time is illustrated in figure 6a where the slope of the linear fit (solid line) corresponds to $3 Z$ thus, $Z=14.0 \pm 0.1$. Knowing $Z$ enables us to calculate the entanglement time for the labelled chain (using the relationship $\tau_{d}=3 Z \tau_{R}$ ) which in our system is in the order of $10^{-3} \mathrm{~s}$. We performed rheology measurements on the polymer L,DPIAA (see Methods Section), (inset of figure 6a). The plateau modulus, $G_{N}^{0}$, was extracted for the UP polymer matrix using the MIN method $\frac{36}{6}$ to yield $G_{N}^{0}=300 \mathrm{~Pa}$. Using the relationship $G_{N}^{0}=\frac{4}{5} \frac{\rho \phi R T}{M_{e}}$ for a polymer solution with $\rho=1600 \mathrm{~kg} / \mathrm{m}^{3}$ and $\varphi=0.003(\varphi$ is the volume fraction of the polymer in solution and $\rho$ the density of the polymer solution), gives $M_{e}=31 \mathrm{~kg} /$ mole where $M_{e}$ is the average molecular weight between topological constraints within a tube $\frac{37}{}$. The polymer average molecular weight $\left(M_{w}\right)$ was measured with AFM and amounted to $380 \mathrm{~kg} / \mathrm{mole}$. The ratio between the average polymer weight and the entanglement molecular weight $\left(M_{e}\right), Z=\frac{5 M_{w}}{4 M_{e}} \frac{35}{5}$ was calculated to be $Z=15$, which is very close to the value $Z=14$ found for the single molecule data. This shows that our approach from the single molecule study in agreement with the rheology experiments.

In figure $6 \mathrm{~b}$ we have plotted the tube diameter $a$ of the polymer chain versus the contour length $L$ of the reptating polymer for a UP matrix consisting of polymers with different molecular weights $\left(M_{w}\right)$ ([Initiator] $/[$ monomer] $=1 / 10,000$ (red round symbols) and 1/2,000 (black square symbols)). As can be seen no correlation is observed since the tube is defined by the density of neighbouring obstacles, which, as long as the matrix polymers are long enough, solely depends on the polymer concentration. In figure 6c both $\tau_{R}$ (left axis, square symbols) and $\tau_{e}$ (right axis, round symbols) are plotted as a function of the contour length $L$. We observed the power laws $\tau_{R}$ and $\tau_{e} \propto L^{2.07 \pm 0.05}$, which is in good agreement with the theory of reptation (the relationship should be with $L^{2}$ ). Additionally, we plotted $\tau_{d}$ as a function of $L$ (Fig. 6d) revealing a power law $\tau_{d} \propto L^{2.9 \pm 0.01}$ in agreement with reptation theory, which predicts $\left(\tau_{d} \propto L^{3}\right)^{1}$. 

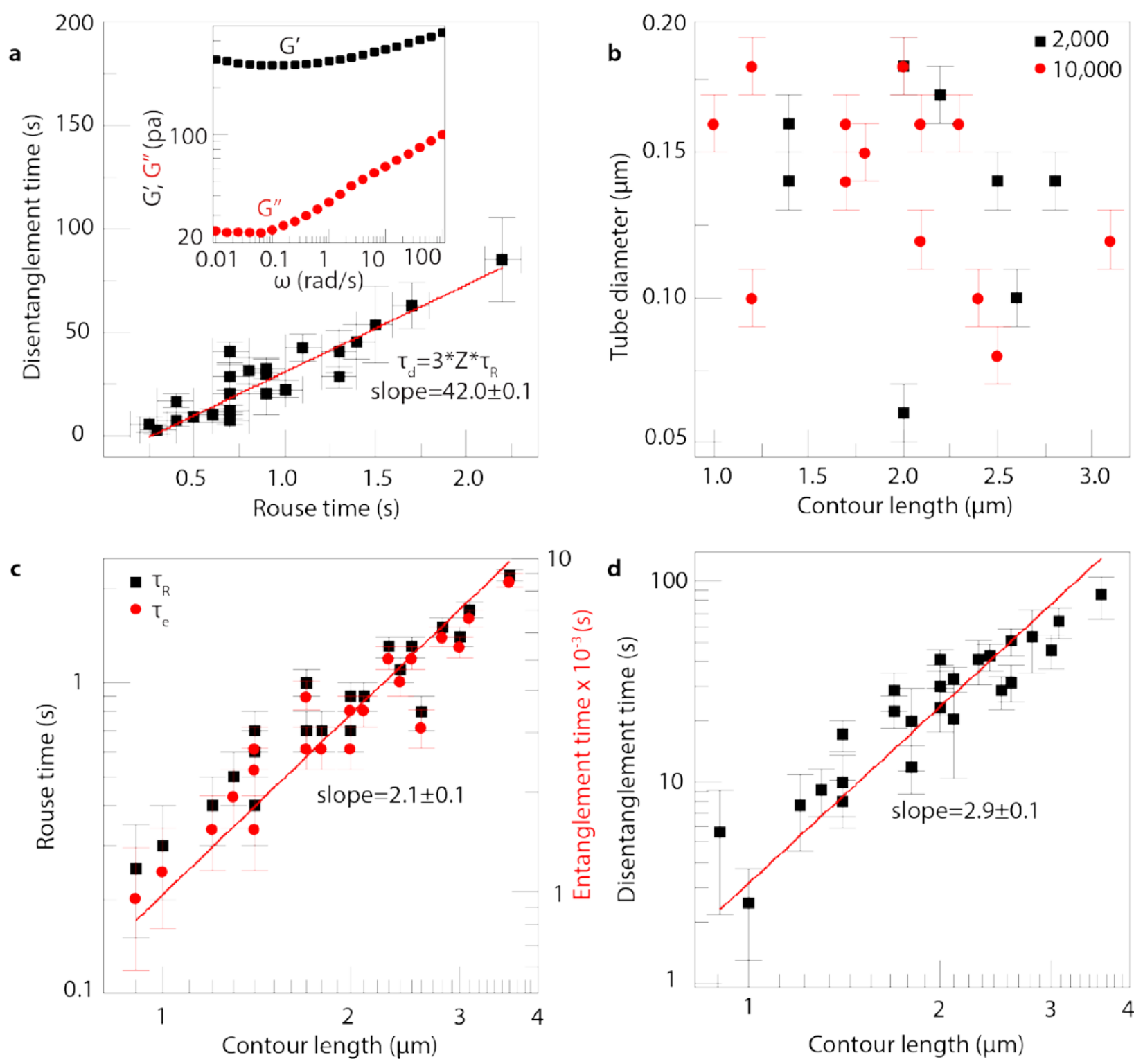

Figure 6| Scaling laws. a, Disentanglement time (tube renewal time) as a function of the Rouse time. The slope of the linear fit is $3 Z$, in agreement with $Z$ extracted from the rheology measurements (see text). Inset: Storage $\left(G^{\prime}\right)$ and loss $\left(G^{\prime \prime}\right)$ modules of the UP matrix L,D-PIAA in tetrachloroethane $(5 \mathrm{mg} / \mathrm{ml})$. b, Tube diameter, $a$, versus contour length, $L$, of the labelled chains (symbols) for polymers of different molecular weight, showing no correlation confirming the fact that the tube diameter depends on the surrounding chains (Ups). No relationship between the tube diameter and molecular weight is observed (see text). c, $\tau_{R}$ and $\tau_{e}$ as a function of contour length $L$ confirming the predictions $\tau_{R}$ and $\tau_{e} \propto L^{2}$. d, Relationship between disentanglement time and contour length. The slope of the curve is in agreement with reptation theory, which predicts $\tau_{d} \propto L^{3}$.

\section{Conclusion}


We have shown that direct visualization of the tube dynamics of a synthetic organic polymer using wide field microscopy is possible. Different analysis methods, which allow one to obtain the tube diameter, have been applied. By carefully analyzing the space- and time-dependence of the dynamics of the polymer chains and using the tube diameter we have probed the local homogeneity/heterogeneity of the polymer matrix. The local reptation and reptation regimes have been observed and the Rouse time has been extracted. Surprisingly, neither the contour length of the reptating chain nor the molecular weight of the surrounding matrix showed any correlation with the tube diameter. We found that the scaling laws as derived from our single molecule experiments are in excellent agreement with De Gennes-Doi-Edwards reptation model. In contrast to biopolymers, which usually have properties that cannot be easily changed, synthetic polymers have the advantage that the parameters of the reptating polymer chains such as the persistence length and the surrounding matrix (material, density, orientation and morphology) can be independently varied. We have demonstrated that with only one experiment all the relevant length and time scales can be extracted. It is good to note that single polymer studies are also in complete agreement the bulk rheology measurement. This approach would allow extracting the same parameters in highly heterogeneous samples, especially crowded cytoskeletons in the cell, as a function of concentration, stiffness, chirality, and type of matrix.

\section{Methods}

Synthesis of polyisocyanides. Poly-isocyano-L-alanyl-D-alanyl methyl ester (L,D-PIAA) was synthesized following the procedure as previously reported $\stackrel{29}{ }$, using 1,1,2,2-tetrachloroethane (TCE) as a solvent and nickel(II) perchlorate as the catalyst. The monomer/catalyst molar ratio was $10,000: 1$ or 2,000:1 depending on the experimental requirement and the as-prepared gelatinous solution was used as the 
matrix for the reptation measurements. The labelled polymer, L-PIAP, was prepared according to an established procedure ${ }^{38}$ with a monomer/catalyst molar ratio of $10,000: 1$ and stored in solution at $4{ }^{\circ} \mathrm{C}$. The polymer was diluted with L,D-PIAA as indicated for the fluorescence measurements. The average polydispersity index (PDI) of polyisocyanides is $1.6-2^{\frac{39}{9}}$.

Rheology measurements. The unlabelled polymer matrix L,D-PIAA was physically characterized as published before $\underline{29}, \underline{30}$. In addition, rheological measurements were performed using a stress-controlled rheometer (Discovery HR-1, TA Instruments) with aluminum parallel plate geometry (40 mm diameter) and a gap of $200 \mu \mathrm{m}$. The storage and loss modulus of the material in the linear regime were obtained by applying an oscillatory strain of $2 \%$ with a frequency range of 0.01 to $100 \mathrm{rad} / \mathrm{s}$ and measuring the sinusoidal stress response at $T=20^{\circ} \mathrm{C}$.

Time-resolved fluorescence microscopy experiments. The motion of the labelled L-PIAP chain was visualized with a wide field microscopy setup. The labelled polymer chains were excited at a wavelength of $543 \mathrm{~nm}$ focused in the back focal plane of a $100 x$ oil objective lens (N.A. $=1.3$, ZEISS). The emission signal was collected with the same objective, separated from the excitation light by a dichroic mirror (DCLP 560) and filtered (LP-565). The movies were recorded with a Princeton Instruments ProEM 512B charge coupled device (ProEM 512B CCD, model: 7555-0005) camera using an integration time of $100 \mathrm{~ms}$. The temporal and spatial resolutions of the experimental setup were $0.1 \mathrm{~s}$ and $200 \mathrm{~nm}$, respectively. Fitting multiple Gaussian point spread functions using MATLAB program allowed us to extract the single chain conformations to a resolution of $\sim 30 \mathrm{~nm}$.

Sample preparation. The samples were prepared by putting a drop of the polymer solution in dichloroethane in between two cover slips, using a perforated Teflon sheet with a thickness of $100 \mu \mathrm{m}$ as a spacer. This closed sample configuration enabled us to limit the evaporation of dichloroethane during the measurement time. 


\section{Acknowledgements}

Financial supports from the Ministry of Education, Culture and Science (Gravity program 024.001.035)

(R.J.M.N., A.E.R.), the European Research Council (ERC Advanced Grant, ALPROS-290886) (R.J.M.N.), the NanoNextNL (7A.06) (A.E.R.), and the NWO Veni Grant (680-47-437) (J.X.) are acknowledged for this work. We further acknowledge the support from HFML-RU/FOM, member of the European Magnetic Field Laboratory (EMFL). Part of this work has been supported by EuroMagNET II under the EU contract number 228043, the Stichting Fundamenteel Onderzoek der Materie (FOM) with financial support from the Nederlandse Organisatie voor Wetenschappelijk Onderzoek (NWO).

\section{Author contributions}

\section{Additional information}

Correspondence and requests for materials should be addressed to M.K., H.E., J.X., J.H., or A.E.R.

\section{Competing financial interests}

The authors declare no competing financial interests.

\section{References}

1 de Gennes, P. G. Reptation of a Polymer Chain in the Presence of Fixed Obstacles. The Journal of Chemical Physics 55, 572, doi:10.1063/1.1675789 (1971).

2 Doi, M. \& Edwards, S. F. Dynamics of concentrated polymer systems. Part 1.-Brownian motion in the equilibrium state. J. Chem. Soc., Faraday Trans. 2 74, 1789-1801, doi:10.1039/F29787401789 (1978). 
Herrmann, A. et al. Mean Square Displacement and Reorientational Correlation Function in Entangled Polymer Melts Revealed by Field Cycling ${ }^{1} \mathrm{H}$ and ${ }^{2} \mathrm{H}$ NMR Relaxometry. Macromolecules 45, 6516-6526, doi:10.1021/ma301099h (2012). Brown, W. \& Zhou, P. Solution Properties of Polyisobutylene Investigated by Using Dynamic and Static Light Scattering and Pulsed Field Gradient NMR. Macromolecules 24, 5151 (1991). Cosgrove, T. \& Sutherland, J. M. Self and mutual diffusion measurements in dilute and semi-dilute polystyrene solutions. Polymer 24, 534-536 (1983).

6 Monkenbusch, M. \& Richter, D. High resolution neutron spectroscopy-a tool for the investigation of dynamics of polymers and soft matter. Comptes Rendus Physique 8, 845-864, doi:10.1016/j.crhy.2007.10.001 (2007).

7 Richter, D. et al. Entanglement constraints in polymer melts. A neutron spin echo study. macromolecules 25, 6156-6164, doi:10.1021/ma00049a011 (1992).

8 Schleger, P., Farago, B., Lartigue, C., Kollmar, A. \& Richter, K. Clear Evidence of Reptation in Polyethylene from Neutron Spin-Echo Spectroscopy. Phys. Rev. Lett. 81, 124-127 (1998).

9 Wischnewski, A. et al. Reptation in polyethylene-melts with different molecular weights. Physica B 276, 337-338 (2000).

10 Kanematsu, T., Sato, T., Imai, Y., Ute, K. \& Kitayama, T. Mutual- and Self-Diffusion Coefficients of a Semiflexible Polymer in Solution. Polymer Journal 37, 65-73, doi:10.1295/polymj.37.65 (2005).

11 Pajevic, S., Bansil, R. \& Konak, C. Dynamic Light Scattering Study of Linear Polyelectrolyte Diffusion in Gels. Macromolecules 28, 7536-7542 (1995).

12 Goetter, R., Kroy, K., Frey, E., Baermann, M. \& Sackmann, E. Dynamic Light Scattering from Semidilute Actin Solutions: A Study of Hydrodynamic Screening, Filament Bending tiffness, and the Effect of Tropomyosin/Troponin-Binding. Macromolecules 29, 30-36 (1996). 
13 Tsunashima, Y., Nemoto, N. \& Kurata, M. Dynamic Light Scattering Studies of Polymer Solutions. 2. Translational Diffusion and Intramolecular Motions of Polystyrene in Dilute Solutions at the theta Temperature. Macromolecules 16, 1184-1188 (1983).

14 Zettl , H. et al. Fluorescence Correlation Spectroscopy of Single Dye-Labeled Polymers in Organic Solvents. Macromolecules 37, 1917-1920, doi:10.1021/ma035929t (2004).

15 Zettl, H., Zettl, U., Krausch, G., Enderlein, J. \& Ballauff, M. Direct observation of single molecule mobility in semidilute polymer solutions. Physical Review E 75, 061804, doi:10.1103/PhysRevE.75.061804 (2007).

16 Zettl, U. et al. Self-Diffusion and Cooperative Diffusion in Semidilute Polymer Solutions As Measured by Fluorescence Correlation Spectroscopy. Macromolecules 42, 9537-9547, doi:10.1021/ma901404g (2009).

17 Liu , R., Gao , X., Adams , J. \& Oppermann, W. A Fluorescence Correlation Spectroscopy Study on the Self-Diffusion of Polystyrene Chains in Dilute and Semidilute Solution. Macromolecules 38, 8845-8849, doi:10.1021/ma0511090 (2005).

18 Texeira, R. E., Dambal, A. K., Richter, D. H., Shaqfeh, E. S. G. \& Chu, S. The Individualistic Dynamics of Entangled DNA in Solution. macromolecules 40, 2461-2476, doi:10.1021/ma062932e (2007).

19 Perkins, T. T., Quake, S. R., Smith, D. E. \& Chu, S. Relaxation of a Single DNA Molecule Observed by Optical Microscopy. science 264, 822-826, doi:10.2307/2884026 (1994).

20 Käs, J., Strey, H. \& Sackmann, E. Direct imaging of reptation for semiflexible actin filaments. nature 368, 226-229, doi:10.1038/368226a0 (1994).

21 Le Goff, L., Hallatschek, O., Frey, E. \& Amblard, F. Tracer Studies on F-Actin Fluctuations. Physical Review Letters 89, 258101, doi:10.1103/PhysRevLett.89.258101 (2002). 
22 Kumaki, J., Kawauchi, T., Yashima, E. \& Yashima. "Reptational" Movements of Single Synthetic Polymer Chains on Substrate Observed by in-Situ Atomic Force Microscopy. Macromolecules 39, 1209-1215, doi:10.1021/ma051933o (2006).

23 Glaser, J. et al. Tube Width Fluctuations in F-Actin Solutions. Physical Review Letters 105, 037801, doi:10.1103/PhysRevLett.105.037801 (2010).

24 Romanowska, M. et al. Direct observation of the tube model in F-actin solutions: Tube dimensions and curvatures. EPL (Europhysics Letters) 86, 26003, doi:10.1209/0295-5075/86/26003 (2009).

25 Nishinaka, T. et al. Visualization of RecA filaments and DNA by fluorescence microscopy. Journal of biochemistry 141, 147-156, doi:10.1093/jb/mvm033 (2007).

26 Smith, D., Perkins, T. \& Chu, S. Self-Diffusion of an Entangled DNA Molecule by Reptation. Physical Review Letters 75, 4146-4149, doi:10.1103/PhysRevLett.75.4146 (1995).

27 Fakhri, N., MacKintosh, F. C., Lounis, B., Cognet, L. \& Pasquali, M. Brownian motion of stiff filaments in a crowded environment. Science 330, 1804-1807, doi:10.1126/science.1197321 (2010).

28 Cornelissen, J. J. et al. beta -Helical polymers from isocyanopeptides. Science 293, 676-680, doi:10.1126/science.1062224 (2001).

29 Cornelissen, J. J. L. M. et al. Synthesis and characterization of polyisocyanides derived from alanine and glycine dipeptides. Journal of Polymer Science Part A: Polymer Chemistry 39, 4255-4264, doi:10.1002/pola.10083 (2001).

30 van Buul, A. M. et al. Stiffness versus architecture of single helical polyisocyanopeptides. Chemical Science 4, 2357, doi:10.1039/c3sc50552a (2013).

31 Schwartz, E. et al. "Helter-skelter-like" perylene polyisocyanopeptides. Chemistry 15, 2536-2547, doi:10.1002/chem.200801746 (2009). 
32 Kouwer, P. H. et al. Responsive biomimetic networks from polyisocyanopeptide hydrogels. Nature 493, 651-655, doi:10.1038/nature11839 (2013).

33 Stephanou, P. S., Baig, C., Tsolou, G., Mavrantzas, V. G. \& Kroger, M. Quantifying chain reptation in entangled polymer melts: topological and dynamical mapping of atomistic simulation results onto the tube model. J Chem Phys 132, 124904, doi:10.1063/1.3361674 (2010).

34 Doi, M. \& Edwards, S. F. The Theory of Polymer Dynamics. (Oxford University Press Inc., 1986).

35 Larson, R. G. et al. Definitions of entanglement spacing and time constants in the tube model. Journal of Rheology 47, 809, doi:10.1122/1.1567750 (2003).

36 Liu, C., He, J., Ruymbeke, E. v., Keunings, R. \& Bailly, C. Evaluation of different methods for the determination of the plateau modulus and the entanglement molecular weight. Polymer 47, 4461-4479, doi:10.1016/j.polymer.2006.04.054 (2006).

37 Huang, Q. et al. Concentrated Polymer Solutions are Different from Melts: Role of Entanglement Molecular Weight. Macromolecules 46, 5026-5035, doi:10.1021/ma4008434 (2013).

38 Schwartz, E. et al. Synthesis, characterisation and chiroptical properties of 'click'able polyisocyanopeptides. Journal of Materials Chemistry 17, 1876, doi:10.1039/b701922j (2007).

39 Schwartz, E., Le Gac, S., Cornelissen, J. J. L. M., Nolte, R. J. M. \& Rowan, A. E. Macromolecular multi-chromophoric scaffolding. Chem. Soc. Rev. 39, 1576-1599, doi:10.1039/B922160C (2010). 